\title{
Interactive comment on "Dynamical downscaling of the western North Pacific from CCSM4 simulations during the last glacial maximum and late 20th century using the WRF model: model configuration and validation" by $\mathrm{J}$. Yoo and $\mathrm{J}$. Galewsky
}

J. Yoo and J. Galewsky

jinwoong.yoo@gmail.com

Received and published: 25 May 2016

Authors thank the anonymous reviewers for their kindness with valuable comments and suggestions. We, here, are trying to provide answers to the comments in a unified fashion. and/or similarities in large-scale atmospheric dynamics and thermodynamics between 
the LGM paleo-environment and the present over the eastern North Pacific domain, from which we are expecting that many interesting research questions can develop. In fact, comparisons between the two geologic time periods can provide many scientific insights to understand the unfolding climate changes in many perspectives including extreme climate events. Authors agree with the reviewers that including these statements would help readers understand the manuscript more clearly.

The CCSM4 LGM simulations are at equilibrium with the prescribed LGM boundary conditions as per PMIP3 protocols. The CCSM4 LGM simulation output that was used in this dynamical downscaling experiment was part of the IPCC-TIER1 CCSM4 Last Glacial Maximum. It is also part of the PMIP3/CMIP5 and is archived with its case name of b40.lgm21ka.1deg.003. The entire CCSM4 LGM simulation was run for one thousand years (Brady et al. 2013). Brady et al. (2013) analyzed the last 30 years of the 1000-year long LGM simulation. Authors used the first 10 years of the last 30 years of the CCSM4 LGM simulation. Please refer to Brady et al. (2013) for further detail of the CCSM4 LGM simulations. 1870 reference year was the year the NCAR scientist started to reproduce the CCSM4 LGM simulation output at the higher temporal resolution of six hour interval. There should not be any issue of equilibrium even with the 1870 reference year for the restart of the simulation but authors wanted to minimize any potential issues if possible. Although ten-year long integration of the dynamical downscaling may not be long enough to fully appreciate the paleoclimate in detail, authors think that even ten-year simulation can suggest a close-to-general condition of the large-scale atmospheric environments at the time of the LGM over the eastern North Pacific domain due to the use of the CCSM4 LGM simulation at equilibrium.

To our best knowledge, there is no paper published for the CMIP5 CCSM4 20th century simulation. However, Brady et al. (2013) can be referred to for further information about the CCSM4 20th century simulation as well. Also, detail description for the CCSM4 1 degree 20th Century Ensemble Member \#6 (MOAR) data (Case Name: b40.20th.track1.1deg.012) can be found at www.cesm.ucar.edu/experiments/cesm1.0.

Interactive comment
Printer-friendly version

Discussion paper

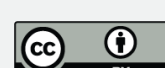


Specifically, the CCSM4 20th century MOAR simulation was run for 1300 years (Brady et al. 2013). The NCAR re-produced 6-hourly outputs for the 20th century (1950-2005) from the 1300 years simulation. Please note that the CCSM4 20th century simulations are NOT at equilibrium because they have been forced with transient greenhouse gases and aerosols (among other things). Authors acknowledge that there is a limitation in the comparison between the 20th century simulation and the LGM simulation because the clearest comparison with the LGM simulations would be with a preindustrial control, which is a true equilibrium climate. Although, we wanted to compare the LGM paleo-environment against the modern condition rather than the preindustrial condition, which would give us more intuitive comparisons with the large-scale atmospheric dynamic and thermodynamic features over the eastern North Pacific.

Regarding the downscaling experiments and the purpose of the manuscript, there are a few things that we would like to make clear. There is no data assimilation applied to our downscaling simulations. It should be understood also that this manuscript is to assess the dynamical downscaling performance of the WRF model as a regional climate model, not to validate the CCSM4 LGM simulations against paleoenvironmental proxy records. For the validation of the CCSM4 LGM simulations, Brady et al. (2013) compared the CCSM4 LGM simulation results of mean annual LGM surface temperature with the Multi-proxy Approach for the Reconstruction of the Glacial Ocean Surface (MARGO) reconstruction (Waelbroeck et al. 2009) and mean annual temperature (MAT) differences from the land-based reconstruction of Bartlein et al. (2011). Additional land-based proxy data from Schmittner et al. (2011), available at http://mgg.coas.oregonstate.edu/;andreas/ data/schmittner11sci/ and those not included in Bartlein et al. (2011), are also utilized for the model validation in Brady et al. (2013), including estimates from ice cores over Greenland and Antarctica. However, since the validation of the CCSM4 LGM simulations has been provided in Brady et al. (2013), comparisons between the GCM (CCSM4 LGM) simulations and the regional downscaling simulations are necessary to evaluate the dynamical downscaling performance of the WRF model as a regional climate model, which is the main purpose

\section{CPD}

Interactive comment
Printer-friendly version

Discussion paper

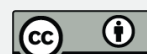


of this manuscript. Please refer to Brady et al. (2013) for the comparisons between the CCSM4 LGM simulation and various proxy records. Nevertheless, authors agree that it will improve our model validation manuscript to compare the downscaling results with the latest reconstruction datasets of paleoenvironment for the LGM such as the MARGO reconstruction data as far as the proxy data exists and observation data for the modern period. Model-to-proxy data and model-to-observation data comparisons would provide valuable information whether downscaling of the CCSM4 using the WRF model performs better than the GCM or not both in the LGM and modern periods.

Reference Brady, E. C., B. L. Otto-Bliesner, J. E. Kay, And N. Rosenbloom, 2013: Sensitivity to Glacial Forcing in the CCSM4, Journal of Climate, 26, 1901-1925.

Interactive comment on Clim. Past Discuss., doi:10.5194/cp-2015-170, 2016. 